\title{
Combining SAR and Optical Data for Environmental Assessments Around Refugee Camps
}

\author{
Andreas Braun and Volker Hochschild \\ Geographisches Institut, Universität Tübingen, Germany·an.braun@uni-tuebingen.de \\ Full paper double blind review
}

\begin{abstract}
This study investigates the combination of images by both radar and optical sensors for environmental modelling in dynamic landscapes. The study area is located in Eastern Kenya and contains three of the largest refugee camps in the world. The fast provision of reliable information about the environmental conditions is needed by humanitarian organizations in order to coordinate their work and help the displaced people. We conducted a land cover classification based on RapidEye, Sentinel-1 and SRTM data using a Random Forest approach. Different combinations of input data were compared in order to achieve best results. The output accuracy was $84 \%$ based on 17 predictor layers. These were generated using second order SAR texture filters and multi-scale topographic indices. Especially a combination of all three input types led to promising results.
\end{abstract}

\section{Introduction}

Political instabilities, terrorism, poverty, harsh climatic conditions and natural disasters force people to migrate throughout all parts of the world. The United Nations High Commissioner for Refugees (UNHCR) counted over 50 million forcibly displaced people at the end of 2013 (UNHCR 2014 $4^{1}$ ). Many of those people gather in refugee camps, which often arise spontaneously and without central control. Humanitarian operations therefore often struggle with the organization of the camp life and their lack of important information. Short-term needs are information on population numbers and camp structure, as well as the distribution of ground water around the refugee camps. In addition, information about the environment and the impacts of the refugee camps on the surrounding resources is critical to both the teams working in the camps (FÜREDER et al. 2014) and regarding the prevention of further migrations (HAGENLOCHER 2011).

Especially in regions where field work is not possible, satellite remote sensing is an important source for fast and reliable information. It can be used for the coordination of help and the assistance for decision-making in order to grant long-term well-being of both the people and the whole region. Our study addresses essential topics for the implementation in humanitarian operations:

Operationalization: Only if the method can be utilized in at least a semi-automated way and within a short time it can provide valuable information in the case of emergency.

GI_Forum - Journal for Geographic Information Science, 1-2015.

(C) Herbert Wichmann Verlag, VDE VERLAG GMBH, Berlin/Offenbach. ISBN 978-3-87907-558-4.

(C) ÖAW Verlag, Wien. ISSN 2308-1708, doi:10.1553/giscience2015s424. 
Transferability: Refugee camps are distributed all over the world. The developed method should not be limited to particular climates or ecosystems.

Data availability: Although this should not be a criterion in the case of emergency, we intended the utilization of freely available datasets and software. In terms of optical data we chose a sensor with high temporal and spatial coverage.

Our study therefore investigates how data can be integrated in a workflow which fulfills the criteria mentioned above. Furthermore, we try to answer the question, which types of data contribute to successful analyses. As many refugee camps lie within regions with different climatic conditions, we additionally explore the benefit of synthetic aperture radar (SAR) data, which penetrates the atmosphere without significant distortions.

\section{Study Area and Input Data}

Our study area (see figure 1) is the region around the refugee camps Dagahaley and Ifo in the North Eastern Province of Kenya. The camps are located around $70 \mathrm{~km}$ from the country border. Together with the refugee camps Hagadera and Kambioos, the Dadaab region hosts a total number of 340.000 refugees, mainly from Somalia, in over 80.000 households (UNHCR 2014 ${ }^{2}$ ). Environmental assessments are important to monitor the changes linked to the camps, which have been established since the civil wars in Somalia in 1991. The climate is semi-arid with two rainy seasons around April and November. The overall precipitation is about $375 \mathrm{~mm}$ per year. At the time of image acquisition (dry season, see Table 1 ), green vegetation cover is limited to shrubs and trees which show considerable activity, while remaining areas are brown and mostly free of grass.
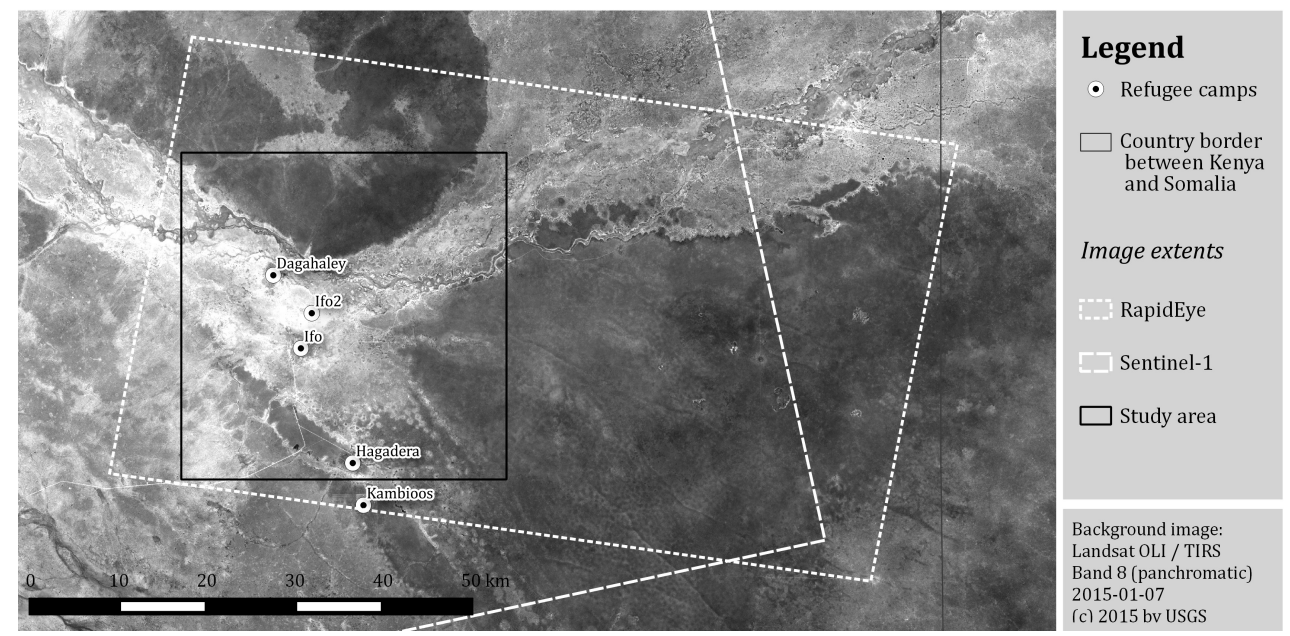

Fig. 1: Location of the study area, refugee camps in the Dadaab region and extents of S1 and RE images. Background: Landsat 8 panchromatic image (C) USGS 
The datasets used in this study are displayed in Table 1. Sentinel-1 (S1) is a C band radar satellite, which was launched within the Copernicus program by the European Space Agency (ESA) in April 2014 (TORRES et al. 2014). The data was purchased as a Level-1 product in Interferometric Wide Swath mode (IW), calibrated to Sigma Naught $\left(\sigma_{\mathrm{db}}^{0}\right)$ and terrain corrected using a range-Doppler algorithm (LOEW \& MAUSER 2007) and a digital elevation model (DEM). In order to preserve the original image textures, no speckle removal was applied. In texture-based classifications the accuracy has been observed to decrease when speckle filtering was applied before classification (PRASAD \& GUPTA 1998, COLLINS et al. 2000). In contrast to L band radar such as ALOS, C band sensors are more sensitive to leaves and small branches, and have a lower penetration depth.

RapidEye (RE) data was purchased as a Level-1 product, orthorectified and atmospherically corrected. The cloud cover of the image is $0 \%$. As shown in Table 1, both images are acquired on the same day granting an optimal phenological comparability. According to TAMSAT data (TARNAVSKY et al. 2014), no rainfall has taken place within the 14 days before the image acquisition. This indicates that the rainy season has already ceased at this time. The radar backscatter of S1 data was therefore not influenced by soil moisture, but predominantly by surface roughness and the water content of plants.

The SRTM 1 ArcSecond digital elevation model (DEM) was released in September 2014 with a spatial resolution of 30 meters (NASA 2014). No further processing was required.

These different sources of information are the basis for using machine learning techniques. As they commonly use a large feature space, several texture parameters have been applied to the SAR image, and a multi-scale terrain analysis has been performed on the DEM (see next chapter).

Table 1: Datasets used in this study

\begin{tabular}{|l|l|l|l|}
\hline Data & Description, spatial resolution & Date & Copyright (Source), Year \\
\hline Sentinel-1 & VV and VH polarization, $10 \mathrm{~m}$ & 27.12 .2014 & ESA (SentHub) 2014 \\
\hline RapidEye & Level-1B product, 5 bands, $5 \mathrm{~m}$ & 27.12 .2014 & BlackBridge AG (EyeFind) 2014 \\
\hline SRTM & 1 ArcSecond, $30 \mathrm{~m}$ & - & USGS (EarthExplorer) 2014 \\
\hline
\end{tabular}

\section{Data Integration and Analysis}

\subsection{Predictor Space Creation}

Classifications based on machine learning techniques are most efficient when a large feature space can be utilized for training and classification. Initially, we had eight layers of information, consisting of two S1 layers (VV and VH polarization), five bands of RapidEye (blue, green, red, red edge, near infrared) and one SRTM layer (elevation in meters). Experimental classifications based on these few layers alone resulted in low accuracies. Additionally, they all cover information based on their spatial resolution alone. In order to utilize the texture information of surfaces, which is revealed within the SAR and the terrain information from SRTM data, we calculated additional input layers for the classification at various scales as described in the following. 


\subsubsection{Sentinel-1}

$\mathrm{S} 1$ data is currently provided in Interferometric Wide Swath mode (IW), with two (VV/VH) polarizations available. We used co-occurrence probabilities, also called Grey Level Cooccurrence matrix (GLCM), defined as "a two dimensional histogram of gray levels for a pair of pixels, which are fixed by a spatial relationship" (HARALICK et al. 1973). It is an acknowledged method to retrieve second order texture information. Orientations of $0^{\circ}, 45^{\circ}$, $90^{\circ}$ and $135^{\circ}$ were used and the pixel offset was set to 1 . The following parameters were calculated using the scikit-image library (VAN DER WALT et al. 2014): Contrast, Correlation, Energy, and Heterogeneity. This increased our feature space based on S1 by the factor 4 . However, we additionally used different window sizes for each texture. As studies have shown that especially small structures exhibit valuable textures for classification (Collins et al. 2000), we chose 3, 5 and 9 pixels as window sizes. This resulted in a total of 12 texture parameters per polarization which could additionally be used for the classification. As these texture layers are redundant to a certain degree, a principal component analysis (PCA) was conducted in order to extract the most valuable variation in the SAR images. Therefore, the 26 SAR parameters ( 1 raw image +12 textures for each of the two polarizations), were reduced to 7 principal components. A comparison of calculations based on all parameters with ones based on only 7 principal components showed that - besides the reduction of input data and computing capacity - there is no significant decrease in the accuracy of the image classification.

\subsubsection{SRTM 30}

A digital terrain analysis was performed on the DEM. By using window sizes of 3, 9 and 45 pixels, the following parameters have been calculated: elevation, slope, aspect and profile curvature. This resulted in 12 additional raster layers for the classification. We experimentally performed a PCA on all SRTM layers as well, but the terrain parameters of smaller window sizes predominantly hindered the creation of homogeneous areas and led to lower classification accuracies. Therefore we excluded those layers from our final calculations.

We did not perform any texture calculations based on the optical images because it turned out to have enough variation to explain landscape patterns. As demonstrated in Table 2, a total of 24 input layers were available as predictor variables for the classification.

Table 2: Datasets used in this study

\begin{tabular}{|l|l|c|}
\hline Data & Layers & Count of Predictor Rasters \\
\hline Sentinel-1 & 7 principal components of 26 texture layers & 7 \\
\hline RapidEye & blue, green, red, red edge, near infra-red & 5 \\
\hline SRTM & 4 terrain parameters with 3 different window sizes & 12 \\
\hline
\end{tabular}

\subsection{Classification}

Machine learning techniques help to detect patterns within ordinal data for a later classification of untrained data according to these detected patterns (ZHANG 2015). In order to make the best use of the radar, optical, and terrain information, we chose a Random Forest (RF) classifier (BREIMAN et al. 2001). It creates a large number of different and uncorrela- 
ted classification and regression trees (CART, BREIMAN et al. 1984). By randomly choosing a subset of training samples and features for the training, the best ruleset is determined, which best splits the feature space into parts, which explain the output attribution of classes. The outputs of every CART are then summed up to a final prediction, in our case a code for land use.

This method has several advantages: (a) although the output map is always a combination of all input layers used, the input data can be of different spatial resolution; (b) different kinds of data can be combined because varying pixel value ranges are allowed, while this can cause problems in other pixel-based methods such as the Maximum Likelihood classifier (FOODY et al. 1992); (c) the landscapes underlie large seasonal dynamics with object dynamics difficult to grasp, whereby a pixel-based approach seems to be appropriate; (d) areas with ambiguous information can be addressed because SAR and optical data are of complementary use for the classification of African landscapes (DEROIN et al. 1998).

For the output generation we chose the Land Cover Classification System (LCCS) suggested by the FAO (DI GREGORIO 2005). It is scale-independent, standardized, and widely approved for mapping purposes. Table 3 lists the classes used for our study area.

Table 3: 10 Land use classes according to the LCC

\begin{tabular}{|l|l|l|}
\hline LCC Code & LCC Label & Remarks \\
\hline $8002-1$ & Perennial Natural Waterbodies (Flowing) & River \\
\hline $8003-1$ & Non-Perennial Natural Waterbodies (Flowing) & River bed \\
\hline $40011-1$ & Open Shrubs & Floodplain \\
\hline $5002-4$ & Paved Roads & Only few \\
\hline $5002-5$ & Unpaved Roads & Most of the paths \\
\hline $5003-14$ & Medium Density Urban Areas & Refugee camps \\
\hline $6005-6$ & Stony Bare Soil Or Other Unconsolidated Materials & Mostly around camps \\
\hline 20055 & Sparse Shrubs & Coverage $<15 \%$ \\
\hline 20017 & Open Shrubs (Shrubland) & Coverage $15-65 \%$ \\
\hline 20021 & Closed Shrubland (Thicket) & Coverage $>65 \%$ \\
\hline
\end{tabular}

In order to train the RF classifier, we manually digitized 150 to 400 random points per class evenly distributed over the study area. In total we used 2500 points for training purposes and additional 500 (30 to 70 per class, depending on their spatial occurrence) for the validation of the result.

\subsection{Workflow}

Figure 2 shows the workflow of our analysis as described in the previous chapters. A subset of the total input data from Sentinel-1 (aggregated into 7 principal components), RapidEye and SRTM was chosen and used to train the classifier. In contrast to simple classification trees, Random Forests do not tend to over-fit the training data, so training accuracies between 40 and $80 \%$ were achieved (see figure 3, dashed line). These were calculated based on the output map and the 2,500 training points it was based on. Additionally, each predictor rasters' importance for the classifier was calculated in order to systematically determine 
the most relevant inputs (see chapter 4). Each subset's classification was validated by the 500 sample points and mapping accuracies were determined. By this we identified the highest possible accuracy.

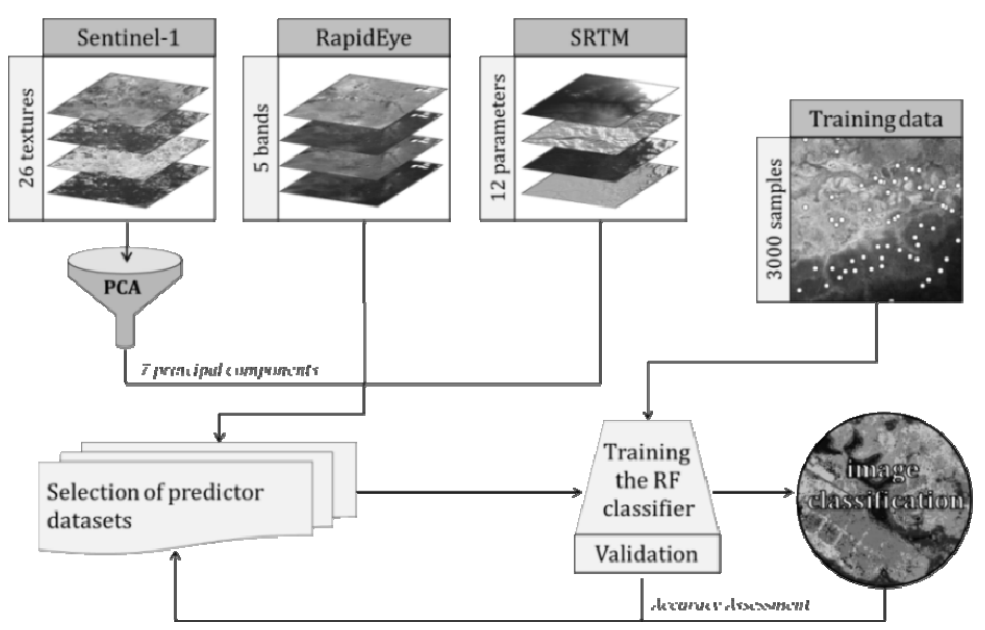

Fig. 2: Workflow for our study. Note the loop between selection of the predictor rasters and image classification to identify the highest classification accuracy.

\section{$4 \quad$ Results and Discussion}

Table 4: Distribution of classes and User's/Producer's and Accuracies of the result.

\begin{tabular}{|l|r|r|r|}
\hline LCC Label & Coverage & UA & PA \\
\hline Perennial Natural Waterbodies (Flowing) & $1,4 \%$ & $86,0 \%$ & $100,0 \%$ \\
\hline Non-Perennial Natural Waterbodies (Flowing) & $10,2 \%$ & $97,7 \%$ & $86,0 \%$ \\
\hline Open Shrubs & $18,6 \%$ & $56,3 \%$ & $90,0 \%$ \\
\hline Paved Roads & $0,2 \%$ & $100,0 \%$ & $100,0 \%$ \\
\hline Unpaved Roads & $1,6 \%$ & $93,9 \%$ & $62,0 \%$ \\
\hline Medium Density Urban Areas & $3,8 \%$ & $97,9 \%$ & $92,0 \%$ \\
\hline Stony Bare Soil Or Other Unconsolidated Materials & $7,6 \%$ & $97,9 \%$ & $97,9 \%$ \\
\hline Sparse Shrubs & $14,9 \%$ & $80,0 \%$ & $48,0 \%$ \\
\hline Open Shrubs (Shrubland) & $15,3 \%$ & $71,4 \%$ & $68,6 \%$ \\
\hline Closed Shrubland (Thicket) & $26,5 \%$ & $80,0 \%$ & $96,0 \%$ \\
\hline
\end{tabular}

The best result has been achieved with the following input layers: 5 RapidEye bands, 7 S1 PCA bands and 5 SRTM layers. In contrast to the S1 layers, SRTM data clearly showed higher importance of the layers with a window size of 45 pixels. Computing 500 Trees resulted in a training accuracy of $77.4 \%$ and an overall classification accuracy of $83.9 \%$. 
Figure 3 demonstrates the stepwise increase of the accuracy by selecting the most important parameters.

Figure 4 shows the final map. Table 4 shows that the classes Non-Perennial Natural Waterbodies, Paved Roads, Medium Density Urban Areas (= refugee camps) and Bare Soil reveal the highest user accuracies, while Open Shrubs (both on the floodplain and as shrubland) were more critical to identify by the input data and the RF algorithm. Regarding producer's accuracy, Perennial Natural Waterbodies and Paved Roads show good results, whereas Unpaved Roads and again classes related to sparse shrub vegetation were of comparatively low accuracy. Altogether, the high accuracies of classes with a high spatial coverage led to a satisfactory overall accuracy of $83,9 \%$.

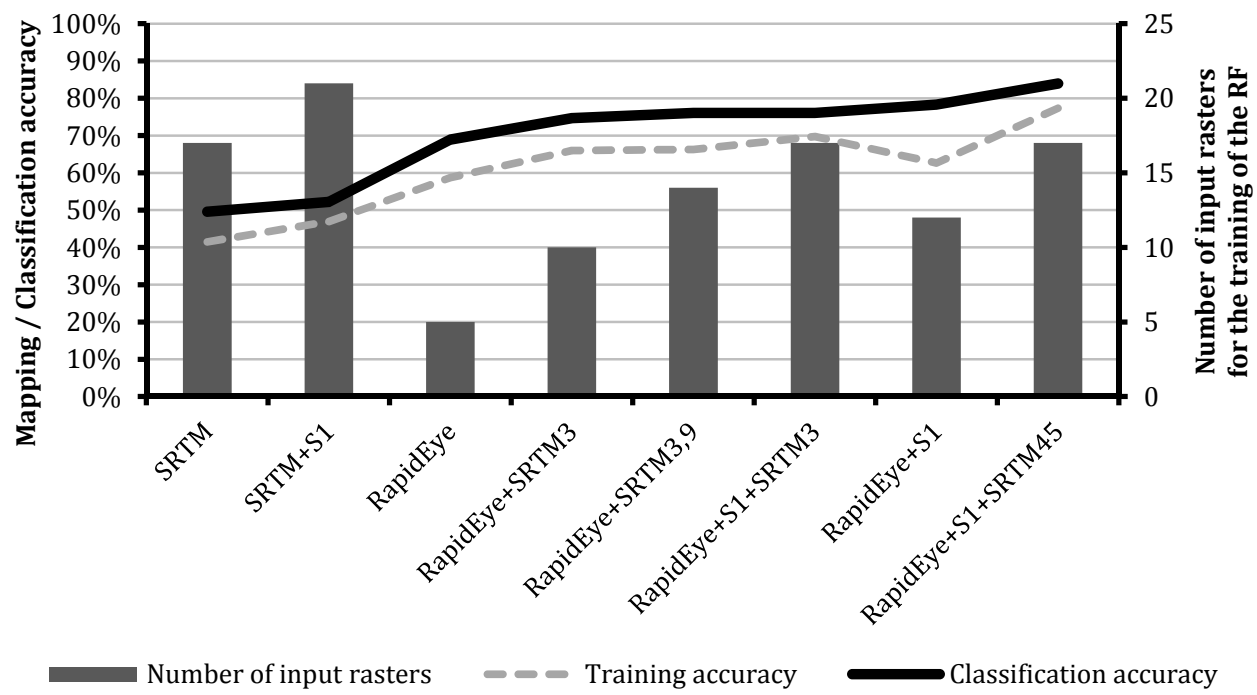

Fig. 3: Accuracies of the trained RF classifier (dashed line) and of the final map (solid line, left y-axis) for selected combinations of predictor rasters (right y-axis). S1: Sentinel-1 PCA Layers, RapidEye: 5 optical bands, SRTM*: Terrain parameters (* The numbers indicate different window sizes for the calculation, see chapter 3.1.2. No number means that all 12 SRTM parameters were used).

Figure 3 contains some more remarkable results: It can be reported that a higher number of input layers (left y-axis) does not necessarily result in better accuracies. As shown by the accuracy of roughly $70 \%$ explained by the RE data alone, it depends on the information content of the predictor layers. First combining optical data with topographic measures and SAR information led to high accuracies and homogenous class areas.

An evaluation of the importance of the different input layers contributing to the creation of the RF and the final classification showed the following results: RapidEye $49 \%$, SRTM $31 \%$ and Sentinel-1 20\%. This indicates that optical information is the most important source of information. The contribution of topographic measures by the SRTM mainly improved the classification of vegetation types at different elevations and landforms. The contribution of Sentinel-1 of only $20 \%$ seems low at first sight but was essential for a dis- 
crimination of optically similar classes such as the refugee camps and floodplains or all types of green vegetation. Furthermore, the combination of RE and S1 showed that even training accuracies of $62 \%$ led to high accuracies in the final classification.

Yet the RF has its weaknesses as well: In contrast to kriging or other geostatistical techniques, it does not take spatial autocorrelation into account. So some small patterns of misclassified areas remain as a typical artefact of randomized trees. These could be extinguished by a mode filter. Class neighbourhoods and spatial trends are surely important for predictions at the landscape scale. Still, as shown in figure 4, the RF still produced large homogenous areas.
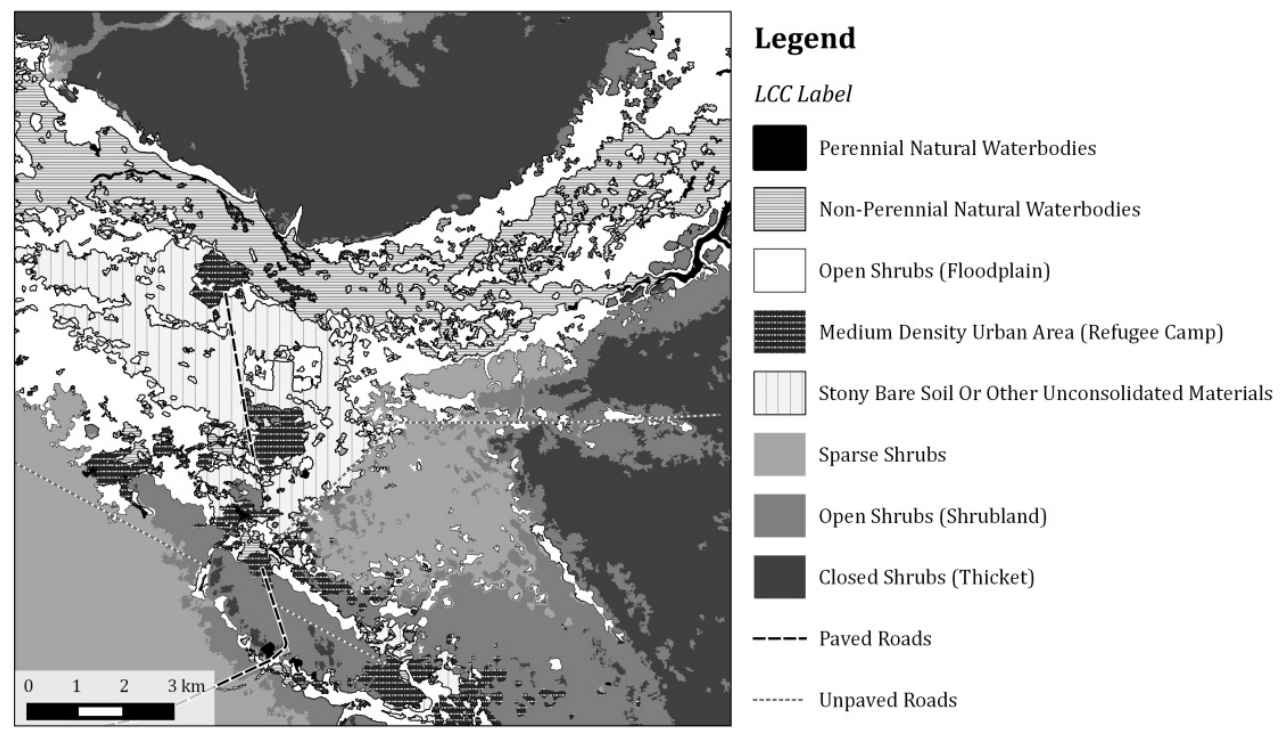

Fig. 4: Result of the classification (output resolution: $7 \mathrm{~m}$ ). The refugee camps are visible as black areas with white dots. A floodplain with a braided river system reaches from NW to NE. Shrubland of different density is located in the North and East. Darker tones indicate thicker shrubs. As the road network is only a few pixels thick it has been digitized manually for the sake of visibility in the map. The patterns in the South are a mosaic of open shrubland, and bare soils caused by seasonal flooding of the plain.

\section{Conclusion and Outlook}

This study showed how datasets of different systems, spatial resolutions, and wavelengths, can be integrated in order to generate high-resolution classifications of dynamic landscapes. Machine learning applications help to find patterns within data, and identify the most valuable information for a later classification. The Random Forest algorithm is independent of scale, and therefore suitable for the application to heterogeneous input data. 
This heterogeneity has been proven to be an asset over the use of a single source as an input for classification. The overall accuracy of $84 \%$ is satisfying, although some of the classes remain difficult to distinguish. While different vegetation cannot be separated by optical data, Sentinel-1 data helped to discriminate between vegetation of different density. Still high errors of commission remain for unpaved roads $(42 \%)$ and open shrubland $(50 \%$, both often confused with bare soil). It is arguable that $\mathrm{C}$ band $(\lambda=3,1 \mathrm{~cm})$ radar is not the optimal wavelength sensitive to biomass. ALOS PALSAR 2 data based on L band $(\lambda=22,9$ $\mathrm{cm}$ ) is not available at present, but could be an option for the future. With reference to elevation information we can report that large scale terrain parameters are of clearly higher value in our classification. The choice of ten land use classes is quite ambitious but required considering the present dynamics. Surely, reducing the overall classes to six or seven could increase the classification accuracy up to over $90 \%$.

The study showed the potential of combined input data for the improvement and support of environmental monitoring. Especially in areas around refugee camps, the development of available biomass and the degradation of soils are of great importance. The use of SAR supports the classification in regions where cloud cover potentially disturbs the interpretation of conventional images. In future studies, we will apply the trained classifier on newly obtained data. As all data are calibrated, change detection is possible. Combinations of RapidEye and Sentinel-1 are still rare, will this will change in the near future when the latter reaches its full operability.

\section{Acknowledgements}

This study was funded by the Austrian Research Promotion Agency (FFG) under the Austrian Space Applications Programme (ASAP 9, Nr. 840081). RapidEye data was kindly provided by BlackBridge AG.

\section{References}

Breiman, L., Friedman, J. H., Olshen, R. A., Stone, C .J. (1984), Classification and Regression Trees. Belmont, Wadsworth, $358 \mathrm{p}$.

BREIMAN, L. (2001), Random Forests. Machine Learning, 45 (1), 5-32.

Collins, M. J., WieBE, J. \& Clausi, D. A. (2000), The effect of speckle filtering on scaledependent texture estimation of a forested scene. IEEE Transactions on Geoscience and Remote Sensing, 38 (3), 1160-1170.

Deroin, J.-P., MotTi, E. \& Simonin, A. (1998), A comparison of the potential for using optical and SAR data for geological mapping in an arid region: The Atar site, Western Sahara, Mauritania. International Journal of Remote Sensing, 19 (6), 1115-1132.

Di Gregorio, A. (2005), Land cover classification system. Classification concepts and user manual. Software version 2. Food and Agriculture Organization of the United Nations, Environment and natural resources series, 8. Rome, 190 p. 
Foody, G. M., Campbell, N. A., Trodd, N. M. \& Wood, T. F. (1992), Derivation and applications of probabilistic measures of class membership from the maximum-likelihood classification. Photogrammetric Engineering and Remote Sensing, 58 (9), 1335 1341.

FÜreder, P., Wendt, L., Hagenlocher, M., Rogenhofer, E. \& LANG, S. (2014), Results of online survey "User requirements for geospatial information products". Issue 1.1. Internal document of the project "EO4HumEn", Z_GIS, Salzburg.

HAgENLOCHER, M. (2011), Assessing the impact of IDP/refugee camps on the state of the environment. An indicator-based approach. In: CAR, A., GRIESEBNER, G. \& STROBL, J. (Eds.), Geospatial crossroads GI_Forum '11. Proceedings of the Geoinformatics Forum Salzburg, Wichmann, Berlin/Offenbach.

Haralick, R. M., Shanmugam, K. \& Dinstein, I. (1973), Textural Features for Image Classification. IEEE Transactions on Systems, Man and Cybernetics, 3 (6), 610-621.

LOEW, A. \& MAUSER, W. (2007), Generation of geometrically and radiometrically terrain corrected SAR image products. Remote Sensing of Environment, 106 (3), 337-349.

NASA (2014), U. S. Releases Enhanced Shuttle Land Elevation Data. National Aeronautics and Space Administration, 08.10.2014. http://www2.jpl.nasa.gov/srtm/ (12/27/2014).

Pedregosa, F., Varoquaux, G., Gramfort, A., Michel, V., Thirion, B., Grisel, O. et al. (2011), scikit-learn: Machine Learning in Python. Journal of Machine Learning Research, 12, 2825-2830.

PERRY, M. (2014), PyImpute: Spatial classification and regression using Scikit-learn and GDAL. Version 0.0.3. https://github.com/perrygeo/pyimpute (1/16/2015).

PRASAD, T. S. \& GUPTA, R. K. (1998), Texture based classification of multidate SAR images - a case study. Geocarto International, 13 (3), 53-62.

Tarnavsky, E., Grimes, D., Maidment, R., Black, E., Allan, R. P., Stringer, M. et al. (2014), Extension of the TAMSAT Satellite-Based Rainfall Monitoring over Africa and from 1983 to Present. Journal of Applied Meteorology and Climatology, 53 (12), 2805 2822.

Torres, R., Snoeij, P., Geudtner, D., Bibby, D., Davidson, M., Attema, E. et al. (2012), GMES Sentinel-1 mission. Remote Sensing of Environment, 120, 9-24.

UNHCR (2014 ${ }^{1}$ ), Global Trends 2013. United Nations High Commissioner for Refugees. www.unhcr.org/statistics (27.12.14).

UNHCR (2014²), Dadaab refugee camps, Kenya. UNHCR bi-weekly update. 16-31 October 2014. United Nations High Commissioner for Refugees.

http://data.unhcr.org/horn-of-africa/download.php?id=1484 (08.01.15).

VAn der Walt, S., Schönberger, J. L., Nunez-Iglesias, J., Boulogne, F., Warner, J. D., YAGER, N. et al. (2014), scikit-image: Image processing in Python. In: PeerJ 2, e453.

ZHANG, Y.-J. (2015), Machine learning for image classification. In: KHOSROw-Pour, M. (Ed.), Encyclopedia of Information Science and Technology. Third Edition. IGI Global, Hershey, PA, USA, 215-226. 